\title{
Sparsity Regularization in Inverse Problems
}

\author{
Bangti Jin \\ Department of Computer Science, University College London, Gower Street, London \\ WC1E 6BT, UK \\ E-mail: b.jin@ucl.ac.uk, bangti.jin@gmail.com
}

\author{
Peter Maaß \\ Zentrum für Technomathematik, Universität Bremen, Fachbereich 03, Postfach \\ 330440, D-28334 Bremen, Germany \\ E-mail: pmaass@uni-bremen.de
}

\section{Otmar Scherzer \\ Computational Science Center, University of Vienna, Oskar-Morgenstern Platz 1, A-1090 Vienna, Austria, and Johann Radon Institute for Computational and Applied Mathematics (RICAM), Austrian Academy of Sciences, Altenbergerstraße 69, A-4040 Linz, Austria \\ E-mail: otmar.scherzer@univie.ac.at}

About two decades ago, the concept of sparsity emerged independently in several disciplines, e.g., statistics [20], signal processing [5] and imaging and inverse problems [19], and has proved to be very successful in a number of applications. The idea is to look for a "sparse" solution whose expansion with respect to a certain basis/frame has many zeros coefficients. Statistically, the sparsity of solutions often leads to better model interpretability and prediction [20], and empirically the sparsity of the signals have been observed. Thus sparsity constraint represents a natural piece of a priori knowledge for solving inverse problems. In the framework of Tikhonov regularization, such a priori knowledge can be effectively enforced by an $\ell^{p}$ penalty on the expansion coefficients. The exponent $p$ in the penalty is often chosen to be $p \in[1,2)$, with the choice $p=1$ especially popular, but a choice $p \in[0,1)$ is also possible.

In 2004, Daubechies, Defrise and De Mol [7] provided a first theoretical treatment on sparsity regularization for ill-posed inverse problems, and established the convergence of an iterative algorithm, i.e., iterative soft thresholding algorithm, for computing regularized solutions. This piece of work has inspired many subsequent developments on sparsity regularization and more general variational regularization (with nonsmooth penalties), and the approach itself has also been established as one of the most powerful tools for solving inverse problems. See the monographs $[16,18]$ and the special issue [17] for many theoretical developments on variational regularization and the survey [10] on sparsity regularization for parameter identification problems. 
Today, sparsity regularization as a paradigm for solving inverse problems has gained much popularity. The aim of this special issue is to provide a forum for ongoing works on this topic. We have been lucky enough to obtain a number of excellent papers, and we would like to thank all contributors for making the special issue possible. The special issue consists of one perspective paper [8] and twelve research papers, with topics covering theoretical developments $[1,13,3,9]$, computational techniques, [6, 11, 21] and novel applications $[4,2,12,14,15]$. Next we give a brief overview of the contributions included in this issue.

In the perspective paper [8], Daubechies, Defrise and De Mol present an overview of early developments of sparsity regularization, including motivations from sparse representation and denoising by thresholding. The perspective centers around the celebrated iterative soft thresholding algorithm, and discusses its derivation, convergence analysis and acceleration techniques. It also contains a complete proof of a previously announced extension of the original algorithm to multiple components and mixed penalties (i.e., multipenalty regularization).

Conventionally, sparsity constraint is enforced by an $\ell^{p}$ penalty in variational regularization. The article [1] studies an alternative formulation for sparse recovery based on discretization, also known as least error method or dual least-square method. The work analyzes the convergence of the method for both a priori and a posteriori rules for choosing the proper discretization level. Further, it establishes sublinear / linear convergence rates under a suitable source condition, and discusses the structure of the source element and approximate solutions.

The issue of choosing an optimal exponent $p$ in the $\ell^{p}$ penalty is scarcely studied in the literature, but nonetheless always fixed in practice. Motivated by parallel MRI, the article [13] explores a "flexible" way of sparse regularization by varying exponents with the index, and gives the proper functional analytic framework, i.e., the so-called $F$-norms instead of the usual setting of normed spaces. One interesting observation is that there are $F$-norms which generate the $\ell^{1}$ space, but they are strictly convex, which contrasts the convex $\ell^{1}$ penalty.

In the absence of a range type source condition and nonlinearity conditions of tangential cone type, convergence rates results for Tikhonov regularization of nonlinear inverse problems are missing in the literature. This is exactly the situation that arises in deautoconvolution in a complex-valued $L^{2}$ space over a finite interval. The article [3] shows that a sparse Fourier representation assumption on the solution implies a variational source condition, which directly yields a convergence rate. Thus surprisingly, the sparsity assumption on the solution enables error estimates, even though the used norm square is not sparsity-promoting.

Practical signals often exhibit multiple desirable features that cannot be adequately described by one single penalty. This calls for more flexible regularization techniques with multiple penalties. The article [9] analyzes optimal support recovery in unmixing with multipenalty regularization, where the regularized functional consists of a datafidelity term, a sparsity-promoting term, and a quadratic penalty term to model the 
noise, and shows that compared with the single-parameter counterpart, the multipenalty approach can significantly enhance support identification.

Inverse problems for partial differential equations (PDEs) are computationally demanding, and there have been intensive activities in leveraging well established techniques in numerical PDEs, e.g., multilevel, adaptive and domain decomposition, to inversion procedures. To estimate the discretization error, the article [6] explores functional error estimators for Tikhonov regularization, which are given in terms of residuals in the optimality system and can be estimated by conventional techniques. A convergence analysis of the resulting adaptive algorithm is provided.

Sparsity regularization for nonlinear inverse problems remain computationally challenging, despite many recent efforts. The works [11, 21] aim at addressing this challenge. The article [11] proposes a variant of the Landweber-Kaczmarz method with inexact solver at each iteration for solving nonlinear inverse problems in Banach spaces using general convex penalty, and analyzes its convergence based on the $\epsilon$-subdifferential calculus. This work improves existing convergence theory, and makes the algorithm more practical. Meanwhile, the article [21] proposes a globally convergent algorithm for computing a minimizer of the Tikhonov-type functional with the $p$-convex $(p \geq 2)$ penalty terms. It employs a dual gradient descent method in the inner iteration, and linearly decreases the regularization parameter in the outer iteration.

Inverse scattering of recovering the shape, locations and medium property from the scattered field underlies many important applications. The article [4] analyzes the resolution and stability properties of sparsity regularization for narrow band array imaging of localized scatterers in several practical scenarios, and discusses reconstruction methods for each scenario. For example, for strong multiple scattering within homogeneous media, it proposes a two-step noniterative method to recover the locations and reflectivities of the scatterers, and to improve its robustness using top singular vectors of the response matrix as optimal illuminations.

Dynamic inverse problems of retrieving time-dependent information from timedependent data are evolving in medical imaging, e.g., PET and SPECT. The article [2] proposes a variational method for simultaneously reconstructing the labelling functions of the subregions and the tracer concentration within each region of dynamic SPECT images. The work provides an analysis of the variational model (e.g., the existence of minimizers and error estimates), a computational algorithm based on alternating direction minimization, and numerical test on synthetic datasets.

Spectral CT using energy-resolved photon-counting detectors can provide the unprecedented material compositions when compared with conventional CT. However, accurate spectral CT needs to account for the detector response function. The article [12] proposes a reconstruction method for spectral CT with a total variation type penalty, i.e., sparse gradient, to take care of the detector response function, which numerically yields more accurate reconstructions than conventional ones.

Image restoration often requires a precise knowledge of the point spread function (PSF). It is spatially variable in large-scale spatial surveys and has to be calibrated 
for postprocessing. The article [14] proposes a resolved component analysis based on matrix factorization to estimate PSFs from a given set of aliased and noisy star images. It exploits the spatial correlation of PSFs across the field of view through sparsity, and is tested on simulated monochromatic PSFs of Euclid telescope.

The article [15] addresses an inverse problem of recovering the scattering and absorption coefficients in a 2D time-dependent radiative transfer equation, which arises in diffuse optical tomography. It discusses the performances and relative merits of three reconstruction algorithms, i.e., sparsity regularization, Landweber-Kaczmarz method, and level set method, in certain practically relevant situations where the diffusion approximation is unsuitable, for either known or unknown layer structures.

Finally, we would like to thank the Inverse Problems team, especially David Jones and Sarah Whitehouse, for their extraordinarily smooth and productive cooperation, as well as Simon R. Arridge for his kind support of our initiative.

\section{References}

[1] Bredies K, Kaltenbacher B and Resmerita E 2016 The least error method for sparse solution reconstruction Inverse Problems 32094001.

[2] Burger M, Rossmanith C and Zhang X 2016 Simultaneous reconstruction and segmentation for dynamic SPECT imaging Inverse Problems 32104002

[3] Bürger S, Flemming J and Hofmann B 2016 On complex-valued deautoconvolution of compactly supported functions with sparse Fourier representation Inverse Problems 32104006

[4] Chai A, Moscoso M and Papanicolaou G 2016 Array imaging of localized objects in homogeneous and heterogeneous media Inverse Problems 32104003

[5] Chen S S, Donoho D L and Saunders M A 1998 Atomic decomposition by basis pursuit SIAM J. Sci. Comput. 20 33-61

[6] Clason C, Kaltenbacher B and Wachsmuth D 2016 Functional error estimators for the adaptive discretization of inverse problems Inverse Problems 32104004

[7] Daubechies I, Defrise M and De Mol C 2004 An iterative thresholding algorithm for linear inverse problems with a sparsity constraint Commun. Pure Appl. Math. 57 1413-57

[8] Daubechies I, Defrise M and De Mol C 2016 Sparsity-enforcing regularisation and ISTA revisited Inverse Problems 32104001

[9] Grasmair M and Naumova V 2016 Conditions on optimal support recovery in unmixing problems by means of multi-penalty regularization Inverse Problems 32104007

[10] Jin B and Maass P 2012 Sparsity regularization for parameter identification problems Inverse Problems 28123001

[11] Jin Q 2016 Landweber-Kaczmarz method in Banach spaces with inexact inner solvers Inverse Problems 32104005

[12] Liu J and Gao H 2016 Material reconstruction for spectral computed tomography with detector response function Inverse Problems 32114001.

[13] Lorenz D and Resmerita E 2016 Flexible sparse regularization Inverse Problems 32 in press

[14] Ngole Mboula F M, Starck J-L, Okumura K, Amiaux J and Hudelot P 2016 Constraint matrix factorization for space variant PSFs field restoration Inverse Problems 32 in press

[15] Prieto K and Dorn O 2016 Sparsity and level Set regularization for diffuse optical tomography using a transport model in 2D Inverse Problems 32 in press

[16] Scherzer O, Grasmair M, Grossauer H, Haltmeier M and Lenzen F 2009 Variational Methods in Imaging (Springer: New York)

[17] Schuster T, Hofmann B and Kaltenbacher B 2012 Tackling inverse problems in a Banach space environment Inverse Problems 28100201 
[18] Schuster T, Kaltenbacher B, Hofmann B and Kazimierski K S 2012 Regularization Methods in Banach Spaces (Walter de Gruyter: Berlin)

[19] Taylor H L, Banks S C and McCoy J F 1979 Deconvolution with the $\ell_{1}$ norm Geophysics 44 39-52

[20] Tibshirani R 1996 Regression shrinkage and selection via the lasso J. Roy. Statist. Soc. Ser. B 58 267-288

[21] Zhong M and Wang W 2016 A global minimization algorithm for Tikhonov functionals with $p$ convex $(p \geq 2)$ penalty terms in Banach spaces Inverse Problems 32104008 